\title{
The Aberrant Course of the Cutaneous Component of the llioinguinal Nerve
}

THOMAS M. OELRICH AND DARVAN A. MOOSMAN

Department of Anatomy, The University of Michigan Medical School, Ann Arbor, Michigan 48109

\begin{abstract}
The ilioinguinal nerve was dissected in 196 adult human cadavers. A normal pattern of distribution, consistent with modern textual descriptions, was defined in $60 \%$ of inguinal regions. In $35 \%$ of the inguinal regions a previously undescribed aberrant course for the cutaneous component of the ilioinguinal nerve was observed in which it is incorporated within the genitofemoral nerve, entered the inguinal canal at the deep inguinal ring, coursed within the cremasteric layer and emerged into the subcutaneous tissue at the superficial inguinal ring on the dorsal side of the spermatic cord or the round ligament of the uterus. The cutaneous component of the ilioinguinal nerve is most frequently found on the ventral surface of the spermatic cord or round ligament during inguinal hernia operations thus an unrecognized aberrant course of this nerve within the spermatic cord is particularly susceptible to accidental trauma.
\end{abstract}

Not infrequently during exposure of the inguinal canal structures, the ilioinguinal nerve cannot be found in its usual or normal position on the ventral aspect of the spermatic cord or round ligament. In such instances, on retraction of the cord or round ligament, a nerve is found dorsal to these structures emerging from the superficial ring and supplying the same cutaneous areas as the normal ilioinguinal nerve. This unusual finding prompted further investigation. The results are reported herein.

The normal ilioinguinal nerve arises from the twelfth thoracic and first lumbar nerve components of the lumbar plexus. It may arise in combination with the iliohypogastric nerve or it may share distal branches as they course between the transverse abdominus and the internal abdominal oblique muscles. The ilioinguinal nerve passes from behind the psoas major muscle and courses obliquely across the quadratus lumborum. It continues along the crest of the ilium or may course obliquely across the iliacus muscle to reach and perforate the transverse abdominus muscle near the anterior superior iliac spine. Here it comes to lie between the transverse abdominus and the internal abdominal oblique muscles where it provides muscular twigs to these muscles. These branches distribute medially supplying the falx inguinalis and communicate with the iliohypogastric and its branches in a plexiform manner. The main ilioinguinal trunk perforates the internal abdominal oblique muscle a few centimeters medial to and slightly below the anterior superior iliac spine, the exact point of emergence is subject to variation (Jamieson et al., '52). In the inguinal region the cutaneous component of the ilioinguinal nerve normally courses between the external abdominal oblique aponeu. rosis and the internal abdominal oblique mus. cle and continues through the inguinal canal lying upon the creamasteric muscle and fascia layer of the spermatic cord or round ligament. The nerve usually enters the subcutaneous tissue as a single nerve in the region of the superficial (clinical synonyms: subcutaneous, medial, external) inguinal ring where it lies on the anterior surface of the spermatic cord (fig. 1). As it enters the subcutaneous tissue the nerve most frequently pierces the medial crus or the external spermatic fascia at the superficial ring. The cutaneous branches of the ilioinguinal nerve distribute to the mons pubis, the thigh (inguinal crease and that portion of the thigh adjacent to the scrotum or labia majora) and the anterior scrotal or

\footnotetext{
Received Nov. 22, '76. Accepted Apr. 29, '77.
} 


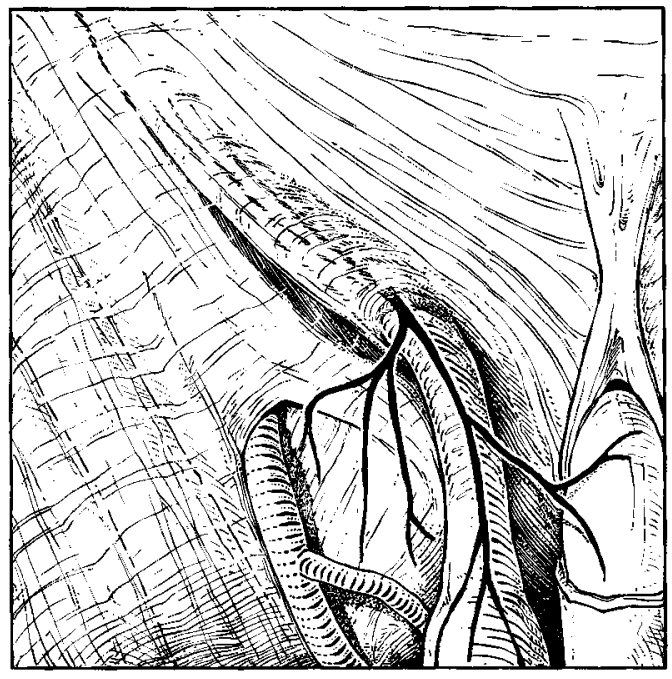

Fig. 1 Normal location of the cutaneous component of the ilioinguinal nerve at the superficial inguinal ring.

labial nerves to the anterior surface of the scrotum or the anterior one-third of the labia majora respectively. There are minor variant points and types of emergence of these normal cutaneous branches (Jamieson et al., '52).

There is, however, one previously undescribed point of emergence which constitutes an aberrant course of the cutaneous component of the ilioinguinal nerve. In this instance this nerve perforates the external spermatic fascia dorsally and laterally and leaves the spermatic cord or round ligament on its dorsal side at the superficial inguinal ring (fig. 2). The cutaneous distribution is unaffected, however, the branches to the pubic region usually pass dorsal to the spermatic cord. In addition to the aberrant placement of this cutaneous component of the ilioinguinal nerve at the superficial inguinal ring, the entire course varies from normal. In these the cutaneous component of the ilioinguinal nerve is found to be in combination with the genitofemoral nerve, which usually arises from $L 1$ and $L 2$ of the lumbar plexus thus sharing L1 with the ilioinguinal nerve. This combined cutaneous component of the ilioinguinal with the genitofemoral nerve follows the usual course of the genitofemoral nerve, perforates the psoas major muscle and lies on its ventral side. The combined nerve resem. bles the genital branch of the genitofemoral nerve, courses lateral to the inferior epigastric artery and enters the deep (clinical syn-

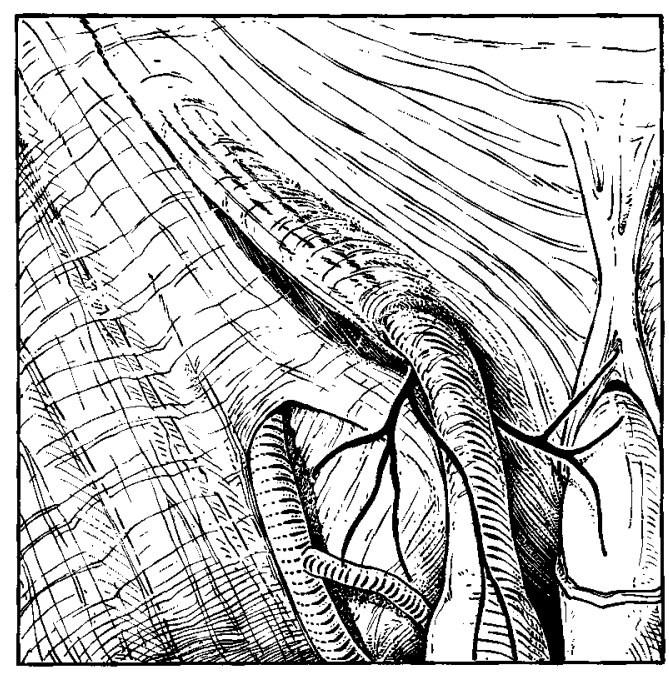

Fig. 2 Aberrant placement of the cutaneous component of the ilioinguinal nerve at the superficial inguinal ring.

onyms: lateral, internal, abdominal) inguinal ring. It then courses through the inguinal canal between the cremasteric layer and the internal spermatic fascia (fig. 3).

Within the inguinal canal the genital branch of the genitofemoral nerve separates to supply the cremasteric muscle, which is its normal distribution. The aberrant ilioinguinal cutaneous component, however, continues medially, lying lateral and inferior to the

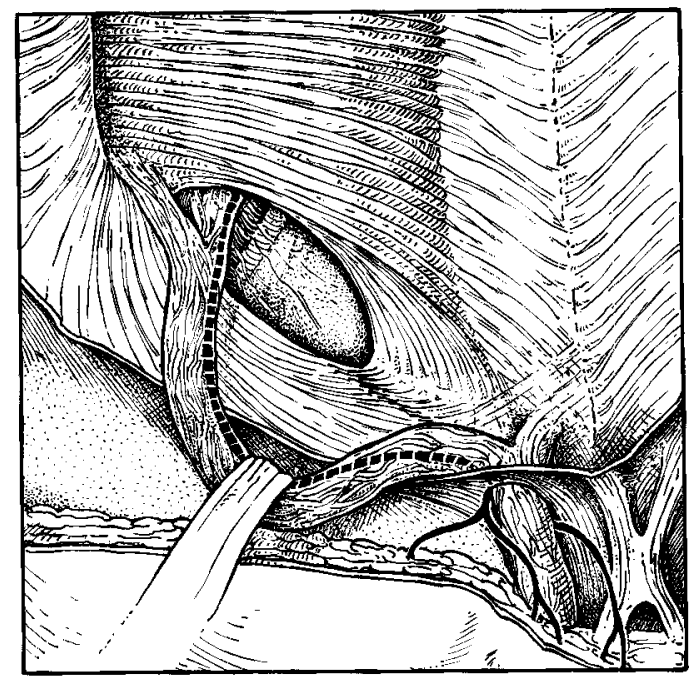

Fig. 3 Aberrant course of the cutaneous component of the ilioinguinal nerve through the spermatic cord. 
TABLE 1

Occurrence of the aberrant course of the cutaneous component of the ilioinguinal nerve

\begin{tabular}{|c|c|c|c|}
\hline & No. of spec. & $\begin{array}{l}\text { Percent of } \\
392 \text { inguinal } \\
\text { areas (sides) }\end{array}$ & $\begin{array}{l}\text { Percent of } \\
196 \text { bodies }\end{array}$ \\
\hline Bodies observed & 196 & & \\
\hline Inguinal areas (sides) observed & 392 & & \\
\hline Left side only & 30 & 7.6 & 15.3 \\
\hline Right side only & 18 & 4.5 & 9.2 \\
\hline \multirow[t]{2}{*}{ Bilateral (right and left) } & 145 bodies $=$ & & \\
\hline & 90 sides 1 & 22.9 & 22.9 \\
\hline Total left side $[45+30]$ & 75 & 19.6 & 38.2 \\
\hline Total right side $[45+18]$ & 63 & 16 & 32.1 \\
\hline Overall occurrence left and right inguinal areas & 138 & 35.2 & \\
\hline
\end{tabular}

spermatic cord, until it reaches the superficial ring where it perforates the cremasteric layer and the external spermatic fascia to enter the subcutaneous tissue. At this point the aberrant component is seen on the dorsal side of the spermatic cord (fig. 2). It divides into the cutaneous branches characteristic of the normal ilioinguinal nerve, namely pubic, scrotal or labial and thigh branches.

In the normal ilioinguinal nerve, muscular branches supply the lower and falx inguinalis fibers of the transverse abdominus and the internal abdominal oblique muscles, however, when its cutaneous fibers join the genitofem. oral nerve, the muscular fibers remain as an independent branch. This muscular component is found in the posterior body wall in the same course and position of the normal ilioinguinal nerve but occasionally the muscular branches may also accompany the iliohypogastric or the lateral femoral cutaneous nerves. In the latter case the muscular branches leave the nerve as it passes beneath the inguinal ligament and recur into the lower border of the transverse abdominus and the internal abdominal oblique muscles. One hundred and ninety-six male cadavers (392 inguinal areas or sides) were observed in laboratory dissection. Thirty-five percent (35\%) of the inguinal areas (sides) demonstrated this aberrant position of the cutaneous component of the ilioinguinal nerve at the superficial inguinal ring. The normal position of the ilioinguinal nerve occurred in $60 \%$ of inguinal areas.

The statistical occurrence of this aberrant course may be reviewed in table 1 . Not all variations in the human body occur in bilateral symmetry. Similarly hernias and hernia repair procedures are usually unilateral. Thus we have chosen to report the occurrence of these aberrant courses relative to inguinal areas or sides.

In 22 inguinal areas (5.6\%) both the normal course and an aberrant course of the cutaneous component of the ilioinguinal nerve were found at the superficial inguinal ring. Here the normal nerve provides the anterior scrotal or labial and pubic branches while the aberrant course supplies the inguinal crease and the thigh.

At the time of this study relatively few female bodies were available. Although the numbers are not adequate for percentage comparison, the types of occurrence indicated for the males in table 1 were also found within the female samples.

\section{DISCUSSION}

The lumbar plexus is the least complex of the major nerve plexuses, however, the resulting nerves are probably the most variable in their composition (Bardeen, '01; Webber, '55). The most variable of its individual nerves are those associated with the abdominal wall musculature. These communicate with adjacent nerves within the layers of muscles and result in complexities of their origin. The combinations of nerves of the lumbar plexus have been recognized for some time, however, their terminal course and distribution has not been clarified. Griffin (1891) suggested that the ilioinguinal nerve might follow the genitofemoral. In some modern texts and atlases this aberrant course of the cutaneous component of the ilioinguinal nerve is illustrated and/or considered incorrectly to be the genitofemoral nerve (Jamieson et al., '52). In fact, the genital branch of the genitofemoral nerve has no cutaneous distribution in and of itself.

The arrangement of the upper branches of 
the lumbar plexus, specifically the combination of the iliohypogastric and ilioinguinal nerves is not unusual and does not affect the course or terminal distribution of these in a significant way. The combination of the ilioinguinal and the genitofemoral nerves in essence subdivides the ilioinguinal into a muscular and a cutaneous component. The cutaneous component accompanies the genitofemoral nerve whereas the muscular component courses independently, resembling the normal ilioinguinal nerve, or may be incorporated in other adjacent nerves. The cutaneous component has a distinctive course or pathway and can be identified by its relation to the spermatic cord (round ligament), and in its manner of emergence from the superficial inguinal ring, in which the normal is found anterior while the aberrant component is found dorsal to the structures.

To prevent postoperative peri-inguinal sensory deficits, during inguinal hernia operations, it is important to identify and preserve both the normal and/or aberrant course of the cutaneous components of the ilioinguinal nerve.

\section{LITERATURE CITED}

Bardeen, C. B. 1901 A statistical study of the abdominal and border-nerves in man. Am. J. Anat., $1:$ 203-228.

Griffin, M. 1891 Some varieties of the last dorsal and first lumbar nerves. J. of Anat., 26: 48-55.

Jamieson, R. W., L. L. Swigart and B. J. Anson 1952

Points of parietal perforation of the ilioinguinal and iliohypogastric nerves in relation to optimal sites for local anaesthesia. Quart. Bull. Northwestern Univ. Med. Sch., 26: 22-26.

Webber, R. H. 1955 Some observations on the constitution of the lumbar plexus of nerves in man. Anat. Rec., 121: 380 . 